\title{
TCF12 activates MAGT1 expression to regulate the malignant progression of pancreatic carcinoma cells
}

\author{
LING WANG ${ }^{1}$, YANJIAO TANG ${ }^{2}$, HONGYI WU $^{3}$ and GUIQIU SHAN ${ }^{4}$ \\ ${ }^{1}$ Department of Clinical Laboratory, The Second Hospital of Shandong University, Jinan, Shandong 250014; \\ ${ }^{2}$ Medical Laboratory, Shenzhen Sami Medical Center, Shenzhen, Guangdong 518038; ${ }^{3}$ Medical Laboratory, \\ Huizhou Municipal Central Hospital, Huizhou, Guangdong 516008; ${ }^{4}$ Department of Transfusion Medicine, \\ General Hospital of Southern Theater Command, Guangzhou, Guangdong 510010, P.R. China
}

Received September 10, 2021; Accepted November 19, 2021

DOI: $10.3892 / \mathrm{ol} .2021 .13180$

\begin{abstract}
As a highly malignant gastrointestinal tumor, pancreatic carcinoma (PC) has poor prognosis due to its low early diagnosis rate, advanced tumor resection and chemotherapy resistance. Magnesium transporter 1 (MAGT1) is a magnesium ion transporter located on the cell membrane, which shows promotive effects on biological behaviors of multiple tumor cells. The aim of the present study was to investigate the role of MAGT1 in the progression of PC and its potential molecular mechanism. Based on the Gene Expression Profiling Interactive Analysis website, MAGT1 was highly expressed in tissues from patients with PC and was associated with poor prognosis. In functional experiments, MAGT1 was highly expressed in PC cell lines. The Cell Counting Kit-8, gap closure and Transwell assays, and western blot analysis, were used to investigate the effects of MAGT1 overexpression or knockdown on the biological behaviors of PC cells. It was found that MAGT1 promoted the proliferation, migration and invasion of PC cells in vitro. According to the Encyclopedia of RNA Interactomes website, transcription factor 12 (TCF12) mRNA expression level was positively correlated with MAGT1 expression level in the tissues from patients with PC. Positive targeting regulation of MAGT1 by TCF12 was also confirmed using a dual-luciferase gene reporter assay and chromatin immunoprecipitation. In addition, knockdown of TCF12 expression inhibited the proliferation and migration of PC cells, but overexpression of MAGT1 expression partly reversed this. These results suggested that TCF12 could promote the proliferation, migration and invasion of PC cells by activating MAGT1 expression, which was associated with
\end{abstract}

Correspondence to: Dr Guiqiu Shan, Department of Transfusion Medicine, General Hospital of Southern Theater Command, 111 Guangzhou Liuhua Road, Guangzhou, Guangdong 510010, P.R. China

E-mail: guiqiushan99@163.com

Key words: pancreatic carcinoma, magnesium transporter 1, transcription factor 12 , poor prognosis poor prognosis. These findings suggest that MAGT1 could be a promising biomarker for the occurrence, progression and prognosis of PC.

\section{Introduction}

Pancreatic cancer (PC) is one of the most lethal types of cancer and the mortality rate ranks second following gastrointestinal tumors worldwide according to cancer statistics in 2018 (1). Pancreatic ductal adenocarcinoma, originating from ductal epithelium, accounts for $\sim 90 \%$ of all cases of PCs, with a 5-year survival rate of $<8 \%$ (2). Despite more advanced diagnostic and treatment methods, the survival rate for PC has not significantly improved. Currently, surgical resection remains the most effective method for the treatment of PC. Unfortunately, most patients are diagnosed with locally advanced or distant metastases from PC and only 15-20\% are eligible for surgical resection $(3,4)$. These results are caused by numerous factors, including its latent nature, rapid progression, low immunogenicity and complex microenvironmental components $(5,6)$. To improve the survival time of the patients and quality of life, the molecular mechanism of PC requires further investigation, and new therapeutic targets.

Magnesium transporter 1 (MAGT1) is a plasma membrane transporter that regulates the basic basal intracellular free magnesium $\left(\mathrm{Mg}^{2+}\right)$ and is selective for the transport of $\mathrm{Mg}^{2+}(7,8)$. MAGT1 has been associated with the development of various malignancies, such as glioma, colorectal cancer and hepatocellular carcinoma (9-13). In colorectal cancer, increased transcription level of MAGT1 was associated with advanced tumor stage, and patients undergoing chemotherapy and with low expression levels of MAGT1 showed longer overall survival time compared with that in patients receiving chemotherapy and with high MAGT1 expression levels (13). Cazzaniga et al (9) revealed that the overexpression of MAGT1 was associated with the drug-resistant phenotype of colorectal cancer. This may be attributed to the abnormal activation of natural killer and CD8 ${ }^{+} \mathrm{T}$ cells caused by MAGT1, which reduces the tumor immune response of $\mathrm{PC}(5,8,14)$. In addition, it has also been found that the effect of Sevoflurane in inhibiting glioma metastasis was achieved by inhibiting cell viability and migration, and promoting apoptosis in glioma cells via the 
circular RNA_0002755/microRNA-628-5p/MAGT1 axis (10). However, there has been little analysis into the role of MAGT1 in the occurrence and progression of PC.

Similar to MAGT1, transcription factor 12 (TCF12) has also been reported to be an oncogene in a variety of cancers, including prostate cancer, gastrointestinal stromal tumors and ovarian cancer (15-18). A study on 120 patients with colorectal cancer showed that patients with high TCF12 expression levels had a higher incidence rate of tumor metastasis and poor survival outcome (19). Chen et al (20) found that TCF12 overexpression in colorectal cancer was regulated by the $\mathrm{CD} 91 / \mathrm{IKK} / \mathrm{NF}-\mathrm{\kappa B}$ signaling cascade and downregulation of E-cadherin increased the incidence rate of metastasis. TCF12 has also been associated with the progression of liver cancer and gastric cancer via the PI3K/AKT, and MAPK/ERK signaling pathways $(21,22)$. In addition, upregulation of TCF12 expression mediated by epigenetic modifications has also been found in melanoma and gallbladder cancer $(23,24)$. However, no reports are available on whether MAGT1 is a downstream target of TCF12.

In the present study, it was hypothesized that MAGT1 may serve as a downstream target of TCF12 to serve a role in PC progression. The present study aimed to investigate whether the TCF12/MAGT1 axis could regulate the proliferation, migration and invasion of PC cells.

\section{Materials and methods}

Bioinformatics analysis. The Gene Expression Profiling Interactive Analysis (GEPIA) website (http://gepia.cancer-pku. $\mathrm{cn} /$ ) was used to analyze the mRNA expression levels of MAGT1 and TCF2 in patients with PC, and to analyze the overall survival and disease-free survival times in patients with PC according to the expression level of MAGT1. The Encyclopedia of RNA Interactomes (ENCORI) website (http://starbase.sysu.edu.cn/) was used to analyze the association between the mRNA expression levels of TCF12 and MAGT1 in PC, and the overall survival time of patients with $\mathrm{PC}$ according to the expression level of TCF12.

Cell culture. The human normal HPDE6c7 pancreatic ductal epithelial cell line and 5 human PC cell lines (SW1990, BxPC-3, PANC-1, CFPAC-1 and AsPC-1) were purchased from American Type Culture Collection. DMEM (Gibco; Thermo Fisher Scientific, Inc.), containing 10\% FBS (Gibco; Thermo Fisher Scientific, Inc.) and $1 \%$ penicillin/streptomycin solution (Beyotime Institute of Biotechnology) was used to culture the cells at $37^{\circ} \mathrm{C}$ in a humidified incubator with $5 \% \mathrm{CO}_{2}$. The cell lines used in the experiment did not pass $>10$ generations.

Cell transfection. The recombinant plasmids, pcDNA3.1MAGT1, pcDNA3.1-TCF12 and the negative control (NC), pcDNA3.1-NC were purchased from Shanghai GeneChem Co., Ltd. MAGT1-specific short hairpin (sh)RNAs (sh-MAGT1-1 and sh-MAGT1-2), non-targeting shRNA, TCF12-specific small interfering (si)RNAs (si-TCF12-1 and si-TCF12-2) and non-targeting siRNA were synthesized, and purified by Guangzhou RiboBio Co., Ltd. Subsequently, $2 \mu \mathrm{g} / \mathrm{ml}$ plasmids, shRNAs and siRNAs were transfected or co-transfected (si-TCF12-2 + pcDNA3.1-MAGT1 or pcDNA3.1-NC) into the BxPC-3 cell line using Lipofectamine ${ }^{\circledR} 3000$ Reagent (Invitrogen; Thermo Fisher Scientific, Inc.) at $37^{\circ} \mathrm{C}$ for $6 \mathrm{~h}$ according to manufacturer's recommendations. Untransfected cells served as an additional control. At $48 \mathrm{~h}$ after transfection, cells were selected for subsequent experiments. The sequences are as follows: sh-MAGT1-1 sense, 5'-GGTCAA ATGTGGAACCATA-3' and antisense 5'-TATGGTTCCACA TTTGACC-3'; sh-MAGT1-2 sense, 5'-GGAGATGGTGTT ATCTGAA-3' and antisense, 5'-TTCAGATAACACCATCTC C-3'; sh-NC sense, 5'-GATCCCCCTTCTCCGAACG-3' and antisense, 5'-AGCTAAAAATTCTCCGAAC-3'; si-TCF12-1 sense, 5'-CAGCAGAGAUACUGGAUUA-3' and antisense, 5'-UAAUCCAGUAUCUCUGCUG-3'; si-TCF12-2 sense, 5'-GGAACAAGUGGUCAACCAA-3' and antisense, 5'-UUG GUUGACCACUUGUUCC-3'; and si-NC sense, 5'-GGCUCU AGAAAAGCCUAUGC-3' and antisense, 5'-CCGAGAUCU UUUCGGAUACG-3'.

$R N A$ preparation, $c D N A$ synthesis, reverse transcriptionquantitative PCR (RT-qPCR). RNA was isolated from cells using MolPure ${ }^{\circledR}$ Cell RNA kit (Shanghai Yeasen Biotechnology Co. Ltd.) in line with the manufacturer's instructions and valuated using a NanoDrop spectrophotometer. RT was performed using a PrimeScript ${ }^{\mathrm{TM}} \mathrm{RT}$ reagent kit (Takara Bio, Inc.) at $42^{\circ} \mathrm{C}$ for $15 \mathrm{~min}$ followed by $85^{\circ} \mathrm{C}$ for $5 \mathrm{sec}$. qPCR was performed three times for each sample using a One Step TB Green ${ }^{\circledR}$ PrimeScript $^{\mathrm{TM}}$ PLUS RT-PCR kit (Takara Bio, Inc.) and the StepOnePlus ${ }^{\mathrm{TM}}$ Real-Time PCR System (Thermo Fisher Scientific Inc.). The thermocycling conditions were as follows: Initial denaturation at $94^{\circ} \mathrm{C}$ for $5 \mathrm{~min}$, denaturation at $94^{\circ} \mathrm{C}$ for $30 \mathrm{sec}$, annealing at $55^{\circ} \mathrm{C}$ for $30 \mathrm{sec}$ and $72^{\circ} \mathrm{C}$ for $30 \mathrm{sec}$ (22 cycles). The mRNA expression level of the target genes was calculated using the $2^{-\Delta \Delta \mathrm{Cq}}$ method (25) and GAPDH was used for normalization. The primer sequences used are as follows: MAGT1 forward, 5'-GGCTTTTGCAGCTTTGTG TT-3' and reverse 5'-AACTGGGCTTGACTGCTTCC-3'; TCF12 forward, 5'-CGTGACCTACTCGGGAATGG-3' and reverse, 5'-CACTTCTAGCAGGTCCGTGG-3' and GAPDH forward, 5'-AATGGGCAGCCGTTAGGAAA-3' and reverse 5'-GCGCCCAATACGACCAAATC-3'.

Western blot analysis. The cells were washed three times with PBS (Corning, Inc.), then they were lysed with pre-chilled lysis buffer (Nanjing SenBeiJia Biological Technology Co., Ltd.) containing protease inhibitor cocktail (Beyotime Institute of Biotechnology) on ice for $10 \mathrm{~min}$, followed by centrifugation at $12,000 \mathrm{x} \mathrm{g}$ for $20 \mathrm{~min}$ at $4^{\circ} \mathrm{C}$. The supernatant was collected and heated $\left(95^{\circ} \mathrm{C}\right.$ for $\left.10 \mathrm{~min}\right)$ together with SDS sample loading buffer (Beyotime Institute of Biotechnology). The proteins were separated using SDS-PAGE, transferred to PVDF membranes (MilliporeSigma), then blocked with TBS (pH 7.6) containing $5 \%$ skimmed milk and $1 \%$ Tween-20 for $1 \mathrm{~h}$ at room temperature. After incubation with the primary antibody overnight at $4^{\circ} \mathrm{C}$, the blots were washed three times with TBS containing $1 \%$ Tween-20 (TBST) and incubated with the corresponding secondary antibody for $1 \mathrm{~h}$ at room temperature. Protein bands were visualized with Chemiluminescent Western Blot Reagents (Thermo Fisher Scientific Inc.) and analyzed using the PDQuest 7.2.0 software (Bio-Rad Laboratories, Inc.). The following antibodies were used: Rabbit anti-MAGT1 
(1:1,000; cat. no. NBP1-69683; Novus Biologicals, LLC); rabbit anti-TCF12 (1:5,000; cat. no. A300-754A; Bethyl Laboratories, Inc.); mouse anti-Ki67 (1:1,000; cat. no. 350502; BioLegend, Inc.); rabbit anti-PCNA (1:1,500; cat. no. LS-B402-50; LifeSpan BioSciences, Inc.); rabbit anti-MMP2 (1:4,000; cat. no. ab92536; Abcam); rabbit anti-MMP9 (1:15,000; cat. no. ab76003; Abcam); rabbit anti-GAPDH (1:2,500; cat. no. ab9485; Abcam), HRP-conjugated goat anti-rabbit IgG H\&L (1:20,000; cat. no. ab6721; Abcam) and HRP-conjugated goat anti-mouse IgG H\&L (1:10,000; cat. no. ab6789; Abcam).

Cell Counting Kit (CCK)-8 assay. Cell proliferation was measured using a CCK-8 assay (Beyotime Institute of Biotechnology). The BxPC-3 cell line was seeded into 96-well plates at a density of $1 \times 10^{4}$ cells per well. After 24,48 and $72 \mathrm{~h}$ of transfection with different plasmids, the medium was discarded. Then, $100 \mu 1$ DMEM containing 10\% FBS and $10 \mu \mathrm{lCCK}-8$ solution were added to each well, and the samples were incubated at $37^{\circ} \mathrm{C}$ for $1 \mathrm{~h}$. The absorbance was measured at $450 \mathrm{~nm}$ using a Multiskan SkyHigh Microplate Spectrophotometer (Thermo Fisher Scientific, Inc.).

Cell migration detection using a gap closure assay. A gap closure assay was performed using culture inserts (Ibidi $\mathrm{GmbH})$. Briefly, control or transfected BxPC-3 cells were cultured in serum-free DMEM in 6-well plates and allowed to reach $70 \%$ confluence. Subsequently, a gap was made using the culture inserts on the surface of the cells and this time was recorded as $0 \mathrm{~h}$. The migration ability of the BxPC- 3 cells was measured by determining the ratio of the gap area $(48 \mathrm{~h})$ to the initial gap area $(0 \mathrm{~h})$ using ImageJ software (version 1.8.0; National Institutes of Health). Images were captured under a light microscope (Olympus Corporation).

Matrigel assay. Transwell chambers (8- $\mu \mathrm{m}$ pore size; Corning, Inc.) were used to determine the invasive ability of the cells. Transfected cells $\left(2 \times 10^{4}\right.$ cells/well) were seeded into the upper chamber pre-coated with $5 \mu 1$ Matrigel $^{\mathrm{TM}}$ (BD Biosciences) and incubated with FBS-free DMEM at $37^{\circ} \mathrm{C}$ for $1 \mathrm{~h}$. A total of $500 \mu \mathrm{l}$ DMEM, containing $10 \% \mathrm{FBS}$, was added to the lower chamber. After incubation at $37^{\circ} \mathrm{C}$ for $24 \mathrm{~h}$, the remaining cells in the upper chamber were removed. Then, the cells that had invaded through the membrane were fixed with $4 \%$ paraformaldehyde (Beyotime Institute of Biotechnology) at room temperature for $10 \mathrm{~min}$. After staining with crystal violet solution at room temperature for $30 \mathrm{~min}$ (Shanghaiyuanye Bio-Technology Co., Ltd), the number of cells passing through the membrane was randomly counted in five fields of view using a light microscope (Olympus Corporation).

Dual-luciferase genereporterassay. The promoter regions from the TCF12 gene were determined using the JASPAR CORE database (http://jaspar.genereg.net/). The wild-type (WT) or mutant (MUT) promoter region of MAGT1 was cloned into the pmir-GLO plasmid (Hunan Keai Medical Equipment Co., Ltd.). MUT MAGT1 was established based on WT MAGT1 using QuikChange Site-Direct Mutagenesis Kit (Agilent Technologies). The mutation sites were located upstream of the MAGT1 transcription start site at -1932 to -1922 (S1) and -162 to $-152(\mathrm{~S} 2)$. A total of $5 \times 10^{4}$ cells per well were seeded into 24-well plates and transfected with WT (S1, 5'-CACAACTGC GC-3' and S2, 5'-CCCACCAGCAA-3') or MUT (S1, 5'-ACA CCAGTATA-3' and S2, 5'-AAACAACTACC-3') MAGT1 and regulatory factors using Lipofectamine ${ }^{\circledR} 3000$ (Invitrogen; Thermo Fisher Scientific, Inc.). At 48 h post-transfection, luciferase activities were analyzed using a Dual-Luciferase Reporter Gene Assay kit (Beyotime Institute of Biotechnology) according to the manufacturer's instructions by comparison with Renilla luciferase activity.

Chromatin immunoprecipitation (ChIP). Formaldehyde solution was added to the cell culture medium to a final concentration of $1 \%$ at room temperature. After fixation for $10 \mathrm{~min}$ at room temperature, glycine was added to terminate the reaction. The cells were washed with pre-cooled PBS and collected in a centrifuge tube for centrifugation $(2,000 \mathrm{x} \mathrm{g}$ for $5 \mathrm{~min}$ for $4^{\circ} \mathrm{C}$ ). The cells were incubated with micrococcal nuclease at $37^{\circ} \mathrm{C}$ for $20 \mathrm{~min}$, then EDTA was added and the samples were centrifuged $(16,000 \mathrm{x} \mathrm{g}$ for $1 \mathrm{~min}$ at $\left.4^{\circ} \mathrm{C}\right)$. After discarding the supernatant, the nuclear pellet was resuspended in ChIP buffer containing protease inhibitor cocktail (Beyotime Institute of Biotechnology), and sonicated (200 Hz; $2 \mathrm{~min}$ ) at $4^{\circ} \mathrm{C}$ to $\sim 500 \mathrm{bp}$. Subsequently, the sheared chromatin was incubated with $10 \mu \mathrm{l}$ TCF12 antibody (dilution, 1:200; cat. no. A300-754A; Bethyl Laboratories, Inc.) overnight at $4^{\circ} \mathrm{C}$. ChIP was performed using a SimpleChIP ${ }^{\circledR}$ Plus Enzymatic Chromatin IP kit (Cell Signaling Technology, Inc.). The $2^{-\Delta \Delta C q}$ method (25) was used to determine the relative fold enrichments and the input chromatin was utilized for the normalization of the samples.

Statistical analysis. All experiments were performed in triplicate. All data were normally distributed after being analyzed with a Shapiro-Wilk normality test. All data are shown as the mean \pm SD. A paired Student's t-test was used for comparisons between tumor and adjacent normal tissues while an unpaired Student's t-test was used for comparison between two groups. Comparisons between more than two groups were evaluated using one-way ANOVA followed by Tukey's post hoc test using GraphPad Prism 8 (GraphPad Software, Inc.).

\section{Results}

MAGT1 mRNA expression level is upregulated in pancreatic carcinoma. To investigate the mRNA expression level of MAGT1 in patients with PC, the mRNA expression level of MAGT1 in patients with PC was analyzed using the GEPIA website. As indicated in Fig. 1A, MAGT1 was highly expressed in tumor tissues from patients with PC. In addition, patients with high MAGT1 expression level also had lower overall survival and disease-free survival times (Fig. 1B and $\mathrm{C}$, respectively). To further determine the protein and mRNA expression level of MAGT1 in PC, western blot analysis and RT-qPCR was performed to analyze the protein and mRNA expression level of MAGT1 in PC cell lines (SW1990, BxPC-3, PANC-1, CFPAC-1 and AsPC-1), and human normal HPDE6c7 pancreatic ductal epithelial cell line, respectively. The PC cells had higher MAGT1 protein and mRNA expression level compared with that in the HPDE6c7 cell line, while the most significant increase in MAGT1 protein and mRNA expression 
A

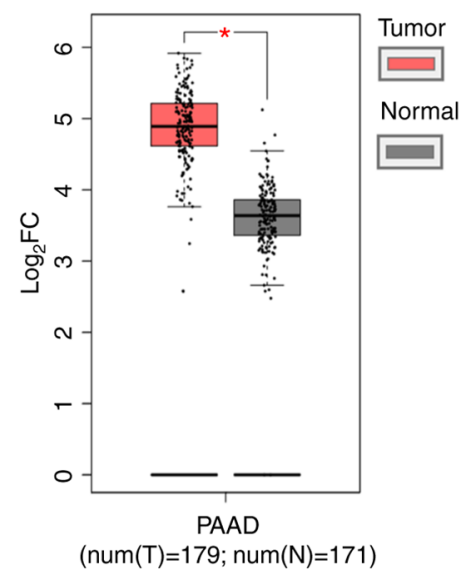

D

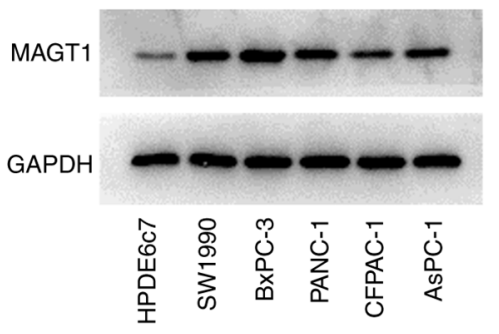

B

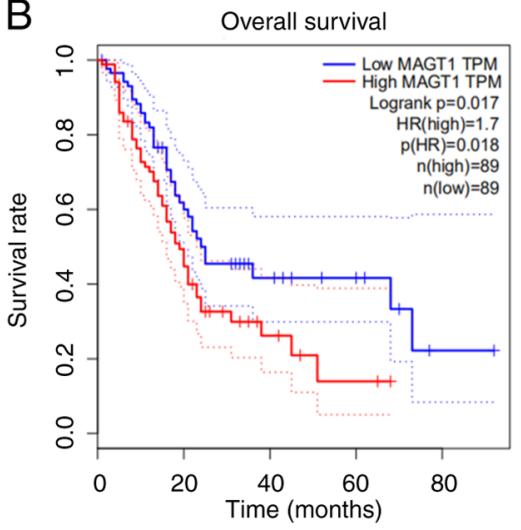

C

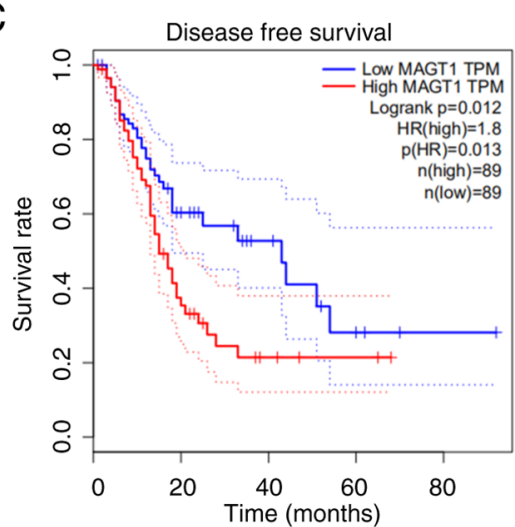

E

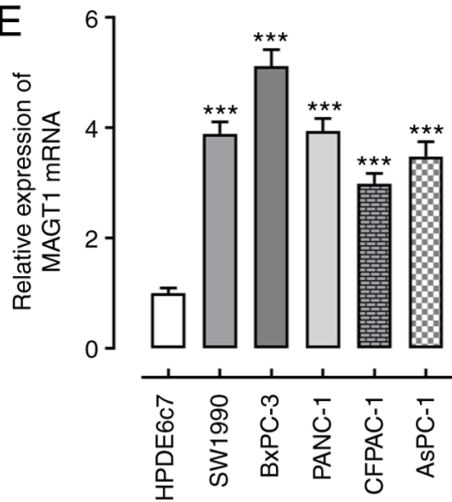

Figure 1. MAGT1 mRNA expression level is increased in PC and is associated with poor prognosis. (A) Gene Expression Profiling Interactive Analysis found that the mRNA expression level of MAGT1 in PC tumor tissues was higher compared with that in normal tissues. Patients with PC and high MAGT1 expression level had shorter (B) overall survival, and (C) disease free survival times. (D) Protein and (E) mRNA expression level of MAGT1 were higher in PC cells (SW1990, BxPC-3, PANC-1, CFPAC-1 and AsPC-1) compared with that in human normal HPDE6c7 pancreatic ductal epithelial cell line. "P<0.05 vs. normal tissues. ${ }^{* *} \mathrm{P}<0.01$ and ${ }^{* * *} \mathrm{P}<0.001$ vs. HPDE6c7 group. T, tumor tissue; $\mathrm{N}$, normal tissue; MAGT1, magnesium transporter 1; PC, pancreatic carcinoma; FC, fold change. PAAD, pancreatic adenocarcinoma.

level was observed in the BxPC-3 cell line (Fig. 1D and E). This suggests that the increase in MAGT1 mRNA and protein expression level was associated with a poor prognosis. In addition, the BxPC-3 cell line was selected for subsequent experiments as it exhibited the highest mRNA and protein expression level of MAGT1.

Overexpression of MAGT1 promotes the proliferation, migration and invasion of pancreatic carcinoma cells in vitro. The BxPC-3 cell line was transfected with MAGT1 overexpression vector, pcDNA3.1-MAGT1 and the NC, pcDNA3.1-NC to investigate the effect of MAGT1 overexpression on cell proliferation, migration, and invasion. Western blot analysis (Fig. 2A) and RT-qPCR (Fig. 2B) confirmed the transfection efficiency of the MAGT1 overexpression vector. In addition, the cells transfected with pcDNA3.1-MAGT1 had a higher level of cell proliferation compared with that in cells transfected with pcDNA3.1-NC (Fig. 2C). The protein expression level of Ki67 and PCNA was also significantly higher in the cells transfected with MAGT1 overexpression vector, which revealed the promotive effect of MAGT1 overexpression on cell proliferation (Fig. 2D). Furthermore, a gap closure assay revealed that after migration for $24 \mathrm{~h}$, the gap area ratio to the initial area in cells transfected with pcDNA3.1-MAGT1 was significantly lower compared with that in cells transfected with pcDNA3.1-NC (Fig. 2E). In addition, a Matrigel assay was also performed and after incubation for $24 \mathrm{~h}$, the cells transfected with pcDNA3.1-MAGT1 had a significantly higher number of invasive cells per visual field compared with that in the cells transfected with pcDNA3.1-NC group (Fig. 2E). The protein expression levels of cell migration markers MMP2 and MMP9 $(26,27)$ were also found to be increased in the cells transfected with pcDNA3.1-MAGT1 compared with that in cells transfected with pcDNA3.1-NC (Fig. 2F). Taken together, these data suggested that overexpression of MAGT1 increased BxPC-3 cell proliferation, migration and invasion in vitro.

Knockdown of MAGT1 expression inhibits the proliferation, migration and invasion of pancreatic carcinoma cells in vitro. Subsequently, the cells were transfected with MAGT1- shRNA (sh-MAGT1-1 and sh-MAGT1-2) or non-targeting shRNA, and untransfected cells served as an additional control. Western blot analysis (Fig. 3A) and RT-qPCR (Fig. 3B) confirmed the knockdown of MAGT1 protein, and mRNA expression level, respectively. In addition, sh-MAGT1-2 was selected for subsequent experiments as it significantly knocked down the expression level of MAGT1-2. As shown in Fig. 3C, there was 
A

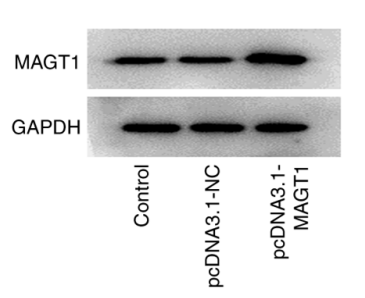

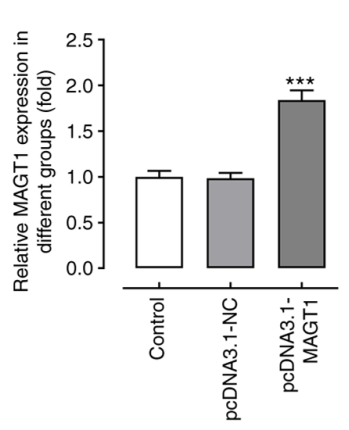
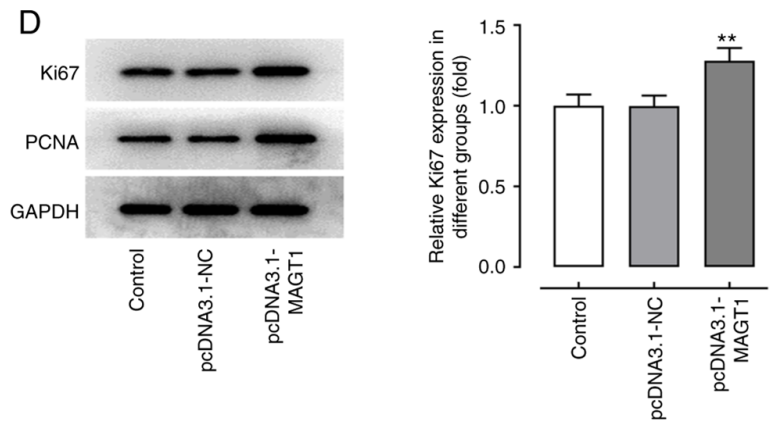

E
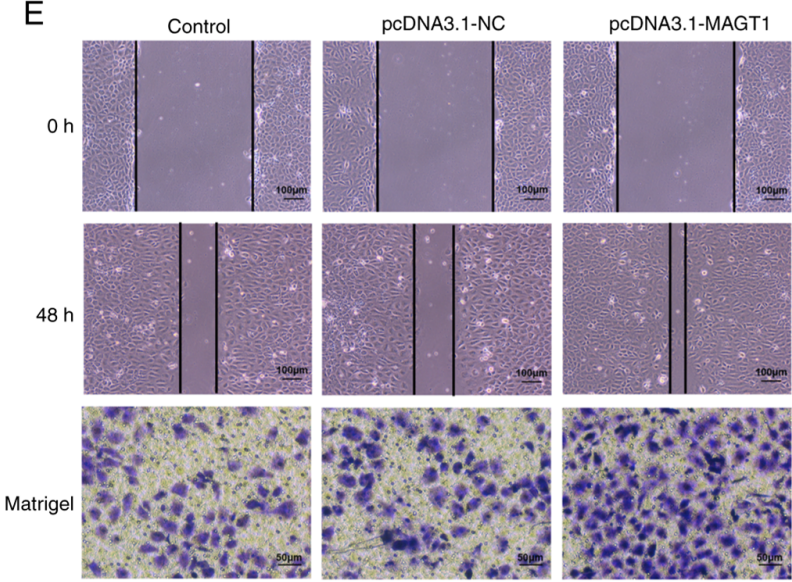

$\mathrm{F}$

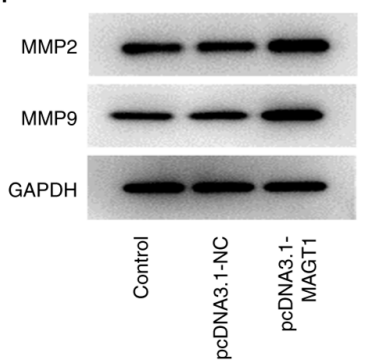

pcDNA3.1-MAGT1
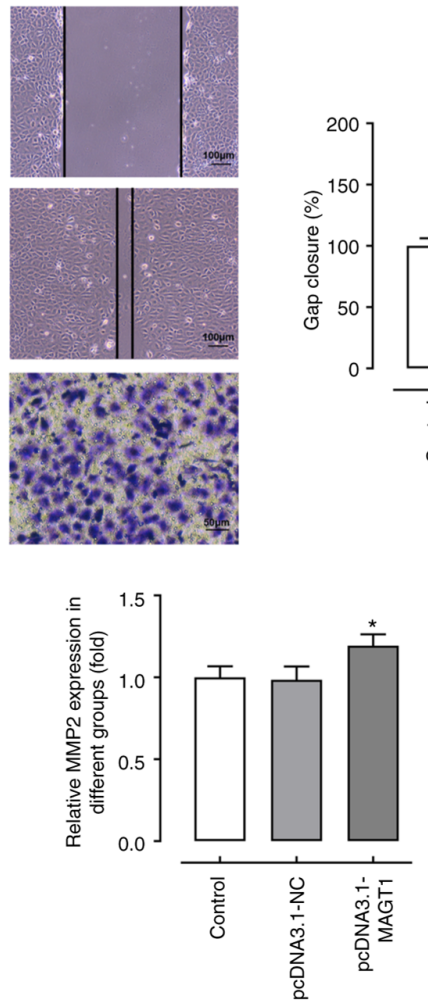

B

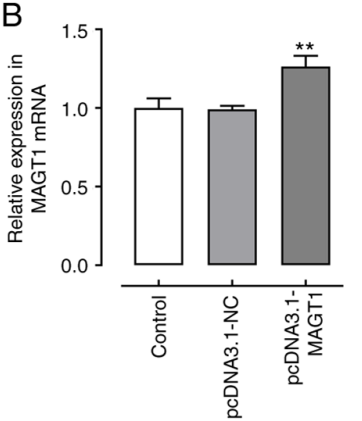

C

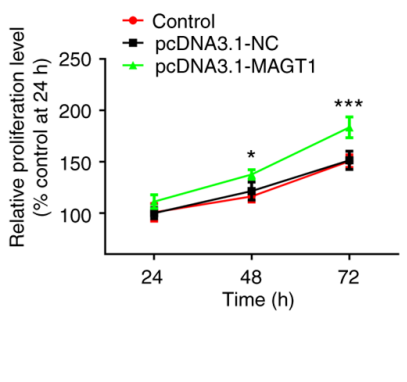

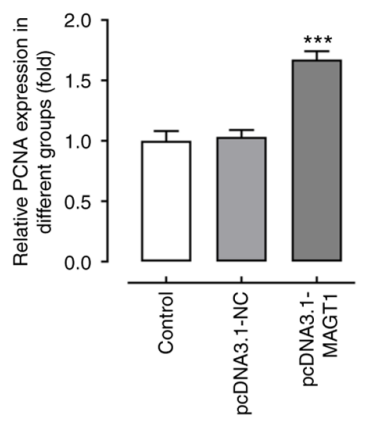
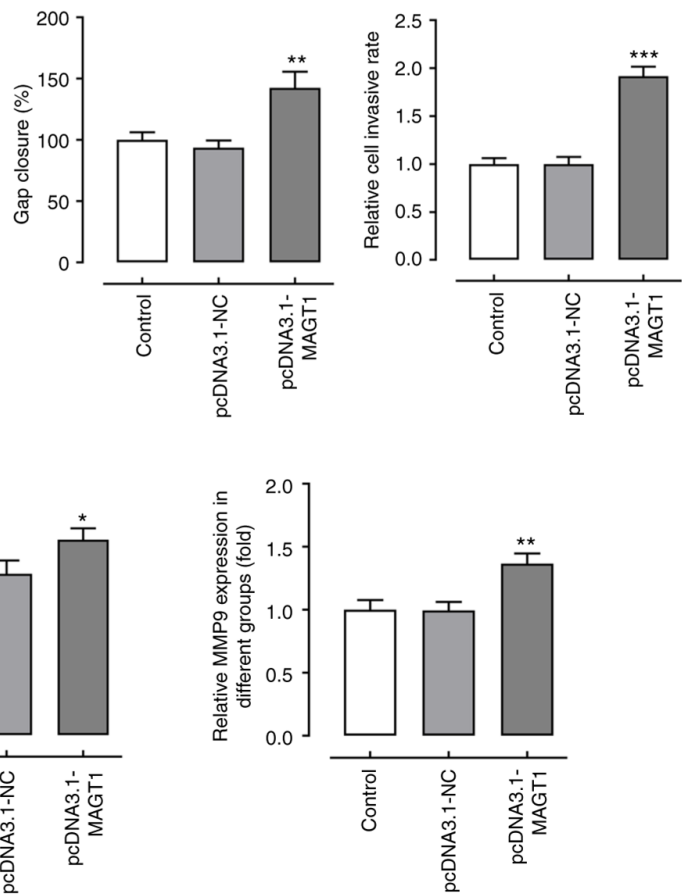

Figure 2. Overexpression of MAGT1 promotes the proliferation, migration and invasion of pancreatic carcinoma cells. (A) Protein and (B) mRNA expression levels of MAGT1 in the BxPC-3 cell line transfected with pcDNA3.1-MAGT1, and pcDNA3.1-NC plasmids using western blot analysis and reverse transcription-quantitative PCR, respectively. Untransfected cells were used as a control. (C) Cell Counting Kit-8 assay was used to analyze cell viability in cells transfected with pcDNA3.1-NC and pcDNA3.1-MAGT1 at different time points (24, 48 and $72 \mathrm{~h}$ ). (D) Representative western blots and quantification showing the protein expression levels of Ki67, and PCNA in the BxPC-3 cells were increased in cells transfected with pcDNA3.1-MAGT1 compared with that in cells transfected with pcDNA3.1-NC. (E) The BxPC-3 cells transfected with pcDNA3.1-MAGT1 had increased cell migration and invasion compared with that in cells transfected with pcDNA3.1-NC. (F) Representative western blots and quantification showing the protein expression level of MMP2, and MMP9 was significantly increased in the BxPC-3 cells transfected with pcDNA3.1-MAGT1 compared with that in cells transfected with pcDNA3.1-NC. "P<0.05, ${ }^{* *} \mathrm{P}<0.01,{ }^{* * *} \mathrm{P}<0.001$ vs. pcDNA3.1-NC group. MAGT1, magnesium transporter $1 ; \mathrm{NC}$, negative control.

a decrease in cell proliferation in the BxPC-3 cells transfected with sh-MAGT1-2 at 24, 48 and $72 \mathrm{~h}$. In addition, the protein expression levels of Ki67 and PCNA are significantly decreased in cells transfected with sh-MAGT1-2 compared with that in cells transfected with sh-NC (Fig. 3D. Furthermore, there was a significant decrease in cell migration and invasion in cells 
A

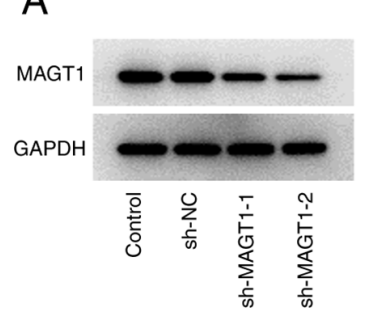

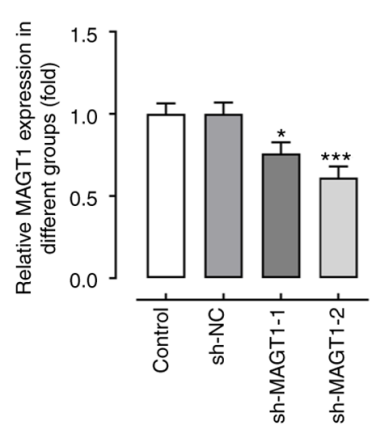

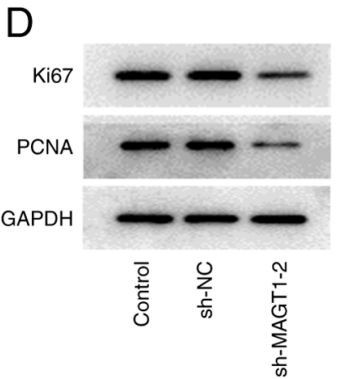

$E$
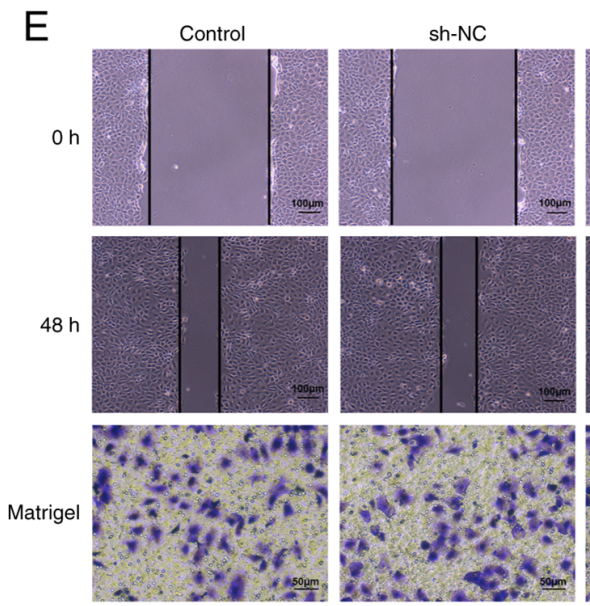

$\mathrm{F}$

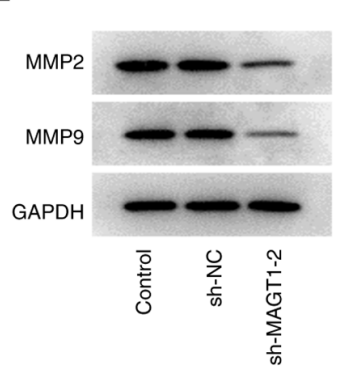

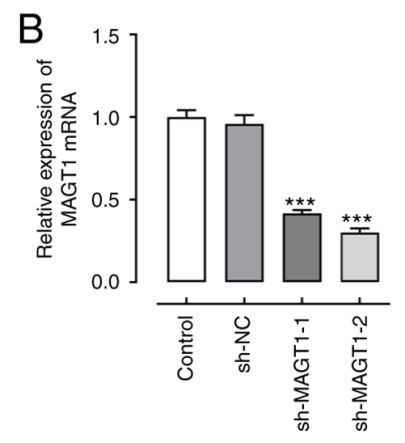

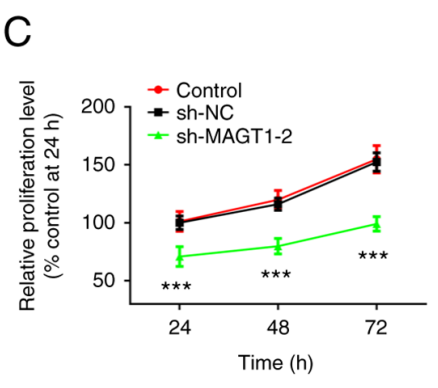

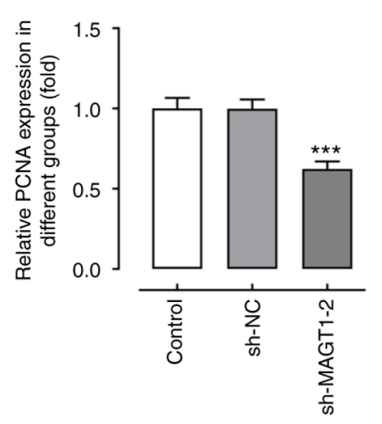
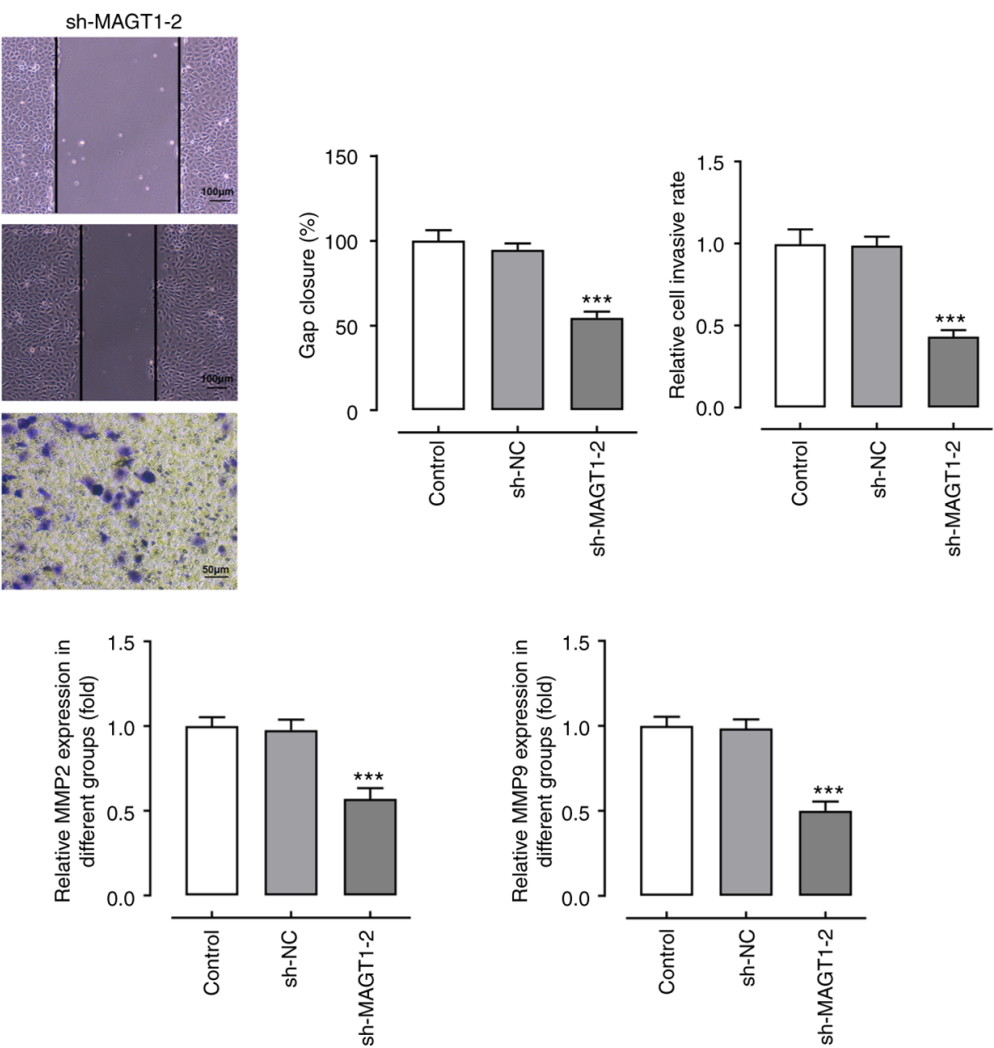

Figure 3. Knockdown of MAGT1 expression inhibits the proliferation, migration and invasion of pancreatic carcinoma cells. (A) Protein and (B) mRNA expression level of MAGT1 in BxPC-3 cells transfected with shMAGT1-1, sh-MAGT1-2 or sh-NC was analyzed using western blot analysis and reverse transcription-quantitative PCR, respectively. Untransfected cells were used as the control. (C) Cell Counting kit- 8 assay was used to analyze cell viability in cells transfected with sh-NC and sh-MAGT1-2 at different time points (24, 48 and $72 \mathrm{~h}$ ). (D) Representative western blots and quantification showing the protein expression levels of Ki67. and PCNA in BxPC-3 cells were decreased in cells transfected with sh-MAGT1-2 compared with that in cells transfected with sh-NC. (E) The BxPC-3 cells transfected with sh-MAGT1-2 had decreased cell migration and invasion compared with that in cells transfected with sh-NC. (F) Representative western blots and quantification showing the protein expression level of MMP2, and MMP9 was significantly increased in the BxPC-3 cells transfected with sh-MAGT1-2 compared with that in cells transfected with sh-NC. ${ }^{*} \mathrm{P}<0.05,{ }^{* *} \mathrm{P}<0.01,{ }^{* * *} \mathrm{P}<0.001$ vs. sh-NC group. MAGT1, magnesium transporter 1; NC, negative control; sh, short hairpin.

transfected with sh-MAGT1-2 compared with that in cells transfected with sh-NC (Fig. 3E). Lastly, the protein expression levels of MMP2 and MMP9 in cells transfected with
sh-MAGT1-2 were also significantly lower compared with that in cells transfected with sh-NC (Fig. 3F). Taken together, these results indicated that knockdown of MAGT1 expression 
A

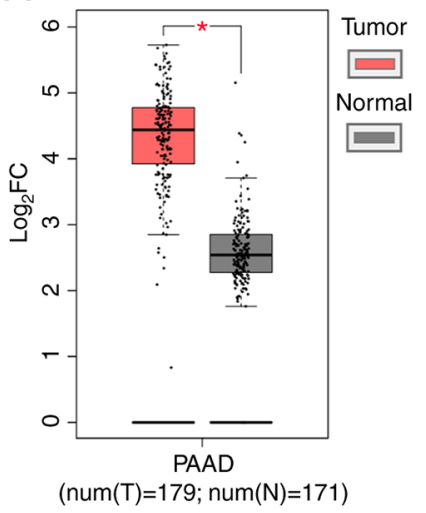

B

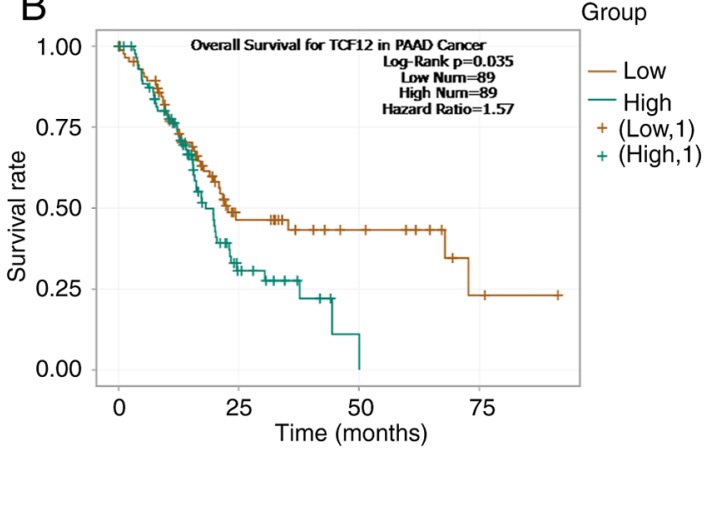

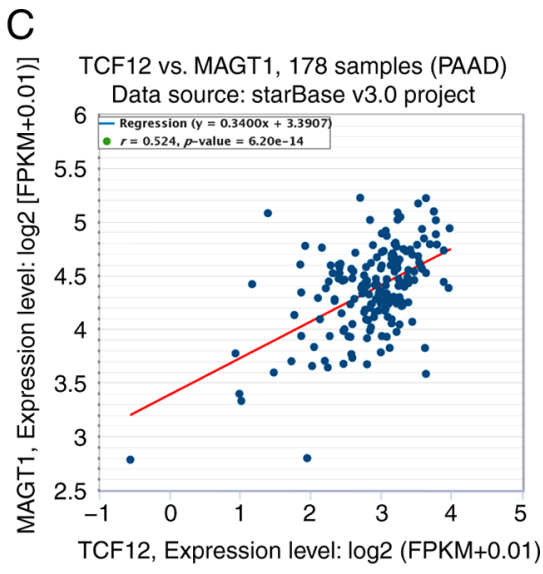

$\mathrm{E}$

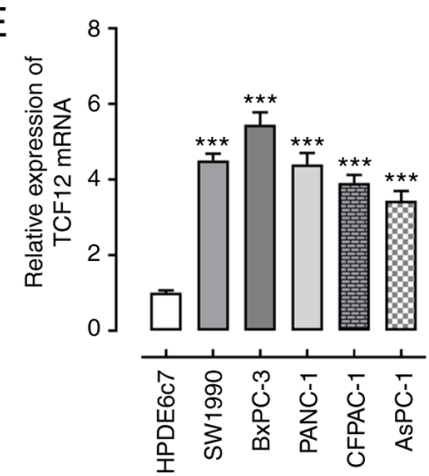

Figure 4. TCF12 mRNA expression level is increased in PC and associated with poor prognosis. (A) Gene Expression Profiling Interactive Analysis revealed that the mRNA expression of TCF12 in PC tumor tissues was higher compared with that in normal tissues. (B) Patients with PC and high TCF12 expression had shorter overall survival time. (C) The results from the Encyclopedia of RNA Interactomes website showed that TCF12 expression level was positively correlated with the expression of MAGT1 in tumor tissues from patients with PC. (D) Protein and (E) mRNA expression levels of TCF12 in PC cells, and human normal HPDE6c7 pancreatic ductal epithelial cell line were analyzed using western blot analysis and reverse transcription-quantitative PCR. * $\mathrm{P}<0.05$ vs. normal tissue. ${ }^{* *} \mathrm{P}<0.01,{ }^{* * *} \mathrm{P}<0.001$ vs. HPDE6c7 group. TCF12, transcription factor 12; PC, pancreatic carcinoma; FC, fold change; T, tumor tissue; $\mathrm{N}$, normal tissue; PAAD, pancreatic adenocarcinoma.

inhibited proliferation, migration and invasion in the $\mathrm{BxPC}-3$ cell line.

TCF12 is positively correlated with MAGT1 expression in pancreatic carcinoma. To investigate the mRNA expression level of TCF12 in patients with PC, the GEPIA and ENCORI databases were used. The GEPIA database showed that mRNA TCF12 expression level was high in tumor tissues from patients with PC (Fig. 4A). In addition, the ENCORI database revealed that patients with high TCF12 expression level had a lower overall survival time (Fig. 4B). There was also a positive correlation between the mRNA expression levels of TCF12 and MAGT1 in the tumor tissues from patients with PC using the ENCORI website (Fig. 4C). Higher TCF12 protein and mRNA expression level was found in the PC cell lines, SW1990, BxPC-3, PANC-1, CFPAC-1 and AsPC-1 compared with that in the HPDE6c7 cell line, in which the BxPC-3 cell line had the most significant increase (Fig. 4D and E). These results suggested that the expression level of TCF12 is increased in PC, was associated with low overall survival time and was positively correlated with MAGT1 expression level.

TCF12 activates MAGT1 expression in the BxPC-3 cell line. The binding sites between TCF12 and the MAGT1 promoter is shown in Fig. 5A. The overexpression and knockdown of TCF12 protein expression was confirmed using western blot analysis, following transfection with pcDNA3.1-TCF12 overexpression vector, and si-TCF12-1 and si-TCF12-2, respectively (Fig. 5B and C). In addition, si-TCF12-2 significantly reduced the protein and mRNA expression level compared with that in cells transfected with si-NC; therefore, it was selected for follow-up experiments. There was increased protein and decreased mRNA expression levels of MAGT1 following overexpression and knockdown of TCF12 expression, respectively (Fig. 5D and E). There was also a significant decrease in luciferase activity following transfection with the plasmids containing the two MUT sequences in MAGT1, S1 and S2 compared with that in cells transfected with the two WT sequences in MAGT1 (Fig. 5F). The aforementioned results indicated that binding of TCF12 to S1 showed stronger activation. Furthermore, the results from a ChIP assay revealed an enriched amplification of fragments from the MAGT1 promoter compared with that in the control using IgG antibodies (Fig. 5G). Taken together, these results suggested that TCF12 could bind to the MAGT1 promoter region and induce MAGT1 expression.

Overexpression of MAGT1 partly reverses the inhibitory effects of TCF12 knockdown expression on the biological behavior of pancreatic carcinoma cells. Cell proliferation in cells transfected with si-TCF12-2 was significantly decreased 
A

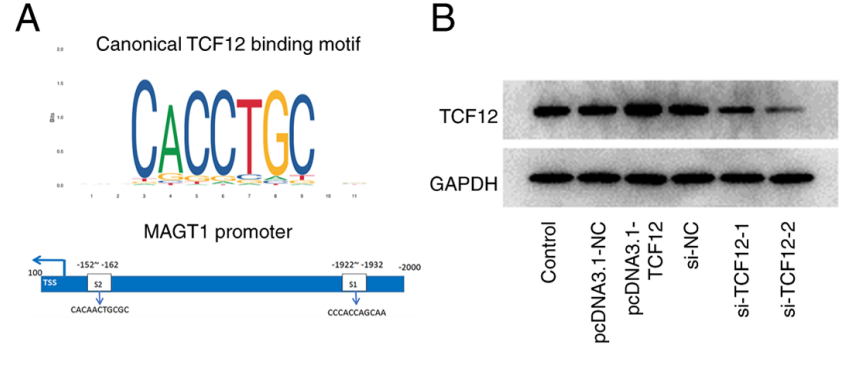

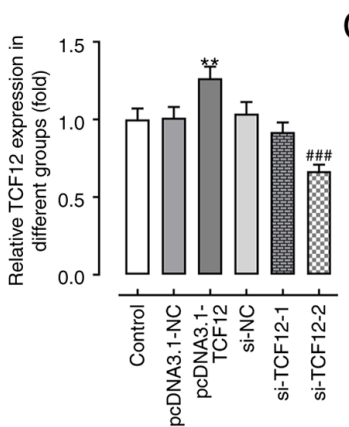

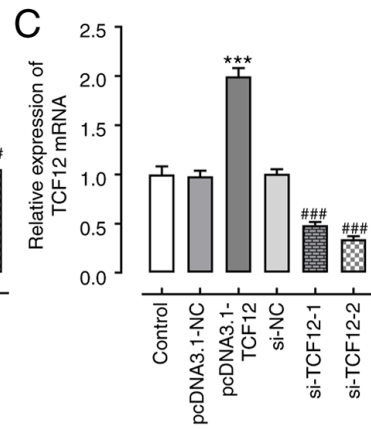

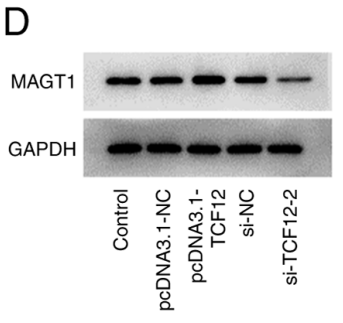

$\mathrm{F}$

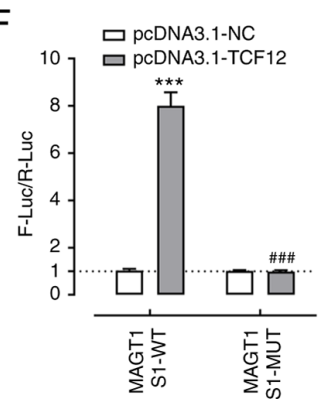

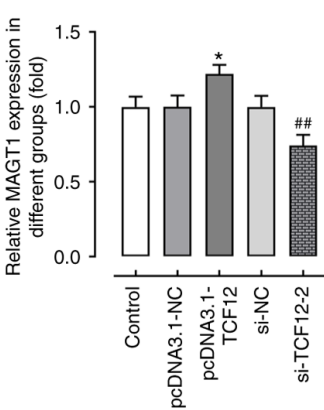

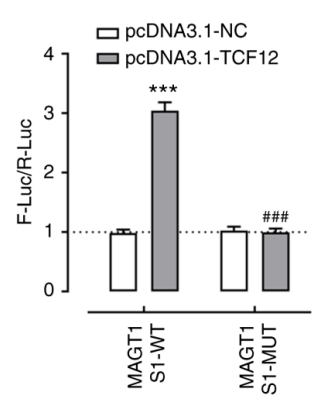

$\mathrm{E}$

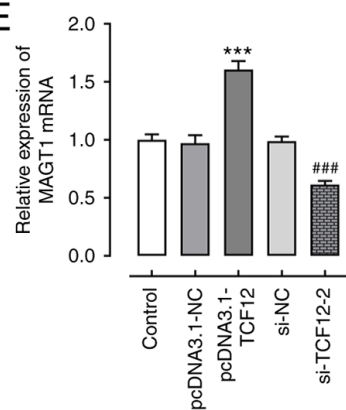

G

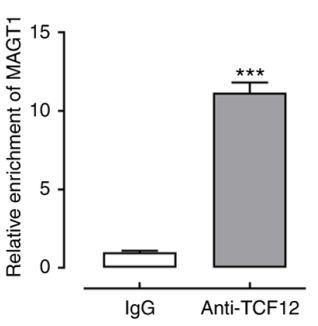

Figure 5. TCF12 binds to the MAGT1 promoter region and induces the expression level of MAGT1 in pancreatic carcinoma cells. (A) The two binding sites of TCF12 on the MAGT1 promoter were predicted using JASPAR. (B) Protein and (C) mRNA expression levels of TCF12 in BXPC-3 cells transfected with pcDNA3.1-TCF12 overexpression vector and pcDNA3.1-NC, and with sh-TCF12-1, sh-TCF12-2 and si-NC were analyzed using western blot analysis and reverse transcription-quantitative PCR, respectively. (D) Protein and (E) mRNA expression levels of MAGT1 in BXPC-3 cells transfected with pcDNA3.1-TCF12 overexpression vector and pcDNA3.1-NC, and with sh-TCF12-1, sh-TCF12-2 and si-NC were analyzed using western blot analysis and reverse transcription-quantitative PCR, respectively. ${ }^{*} \mathrm{P}<0.05,{ }^{* *} \mathrm{P}<0.01,{ }^{* * * *} \mathrm{P}<0.001$ vs. pcDNA3.1-NC group; ${ }^{\# \#} \mathrm{P}<0.01,{ }^{\# \# "} \mathrm{P}<0.001$ vs. si-NC group. (F) Luciferase assay revealed an increase in the activity of the MAGT1 promoter in BxPC-3 cells after co-transfection with the TCF12 expression plasmid. The activity of the MAGT1 promoter is higher after S1 is combined. The activity of the lysate from cells co-transfected with the plasmid containing the TCF12 promoter fragment and pcDNA3.1-NC was considered $100 \%$. ${ }^{* * *} \mathrm{P}<0.001$ vs. pcDNA3.1-NC + MAGT1 S1-WT group; ${ }^{\# \# ~} \mathrm{P}<0.001$ vs. pcDNA3.1-TCF12 + MAGT1 S1-WT group. (G) Chromatin immunoprecipitation revealed an enrichment of amplified fragments from the MAGT1 promoter in the anti-TCF12 group using IgG antibodies as the control. ${ }^{* * *} \mathrm{P}<0.001$ vs. IgG group. TSS, transcription start site; TCF12, transcription factor 12; MAGT1, magnesium transporter 1; NC, negative control; WT, wild-type; MUT, mutant; si, small interfering.

at 24,48 and $72 \mathrm{~h}$ post-transfection compared with that in cells transfected with si-NC (Fig. 6A). In addition, in the BxPC-3 cells transfected with si-TCF12-2 and MAGT1 overexpression plasmid there was increased cell viability. Western blot analysis also showed reduced protein expression levels of Ki67 and PCNA in cells transfected with si-TCF12-2 compared with that in cells transfected with si-NC. There was also increased protein expression level in cells transfected with si-TCF12-2 and pcDNA3.1-MAGT1 compared with that in cells transfected with si-TCF12-2, and pcDNA3.1-NC. This suggests that overexpression of MAGT1 reversed the decreased viability of the BxPC-3 cells induced by knockdown of TCF12 gene expression (Fig. 6B). As shown in Fig. 6C, $24 \mathrm{~h}$ following transfection with si-TCF12-2, the gap closure was significantly higher in cells transfected with si-TCF12-2 compared with that in cells transfected with si-NC. However, after co-transfection with pcDNA3.1-MAGT1 and si-TCF12-2, the gap closure was partially reversed. Similarly, the protein expression levels of MMP2 and MMP9 were significantly lower in cells transfected with si-TCF12-2 compared with that in cells transfected with si-NC. The protein expression levels increased in cells transfected with si-TCF12-2 and pcDNA3.1-MAGT1 compared with that in cells transfected with si-TCF12-2, and pcDNA3.1-NC (Fig. 6D). Taken together, these results suggested that MAGT1 could be a functional gene of TCF12. Knockdown of TCF12 expression could inhibit the proliferation, migration and invasion of PC cells, while these effects could be partly reversed by upregulating the expression level of MAGT1.

\section{Discussion}

$\mathrm{PC}$ is one of the most aggressive types of cancer affecting human health worldwide (2). An essential regulator of PC is the MAGT1. MAGT1, as a major mechanism of $\mathrm{Mg}^{2+}$ influx, 
A

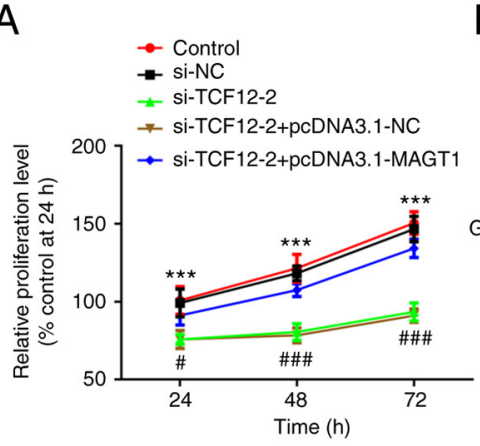

B

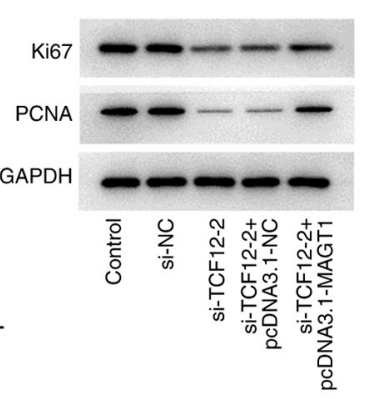

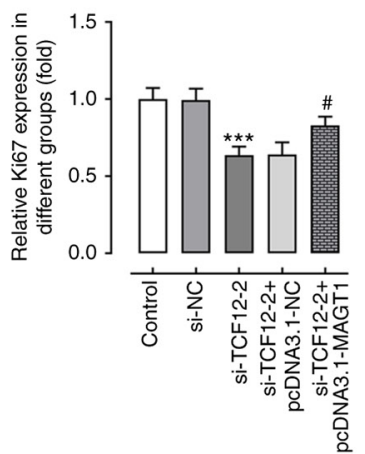

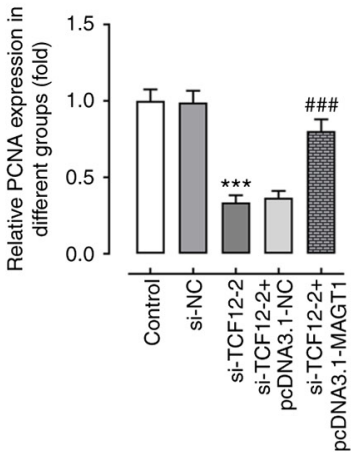

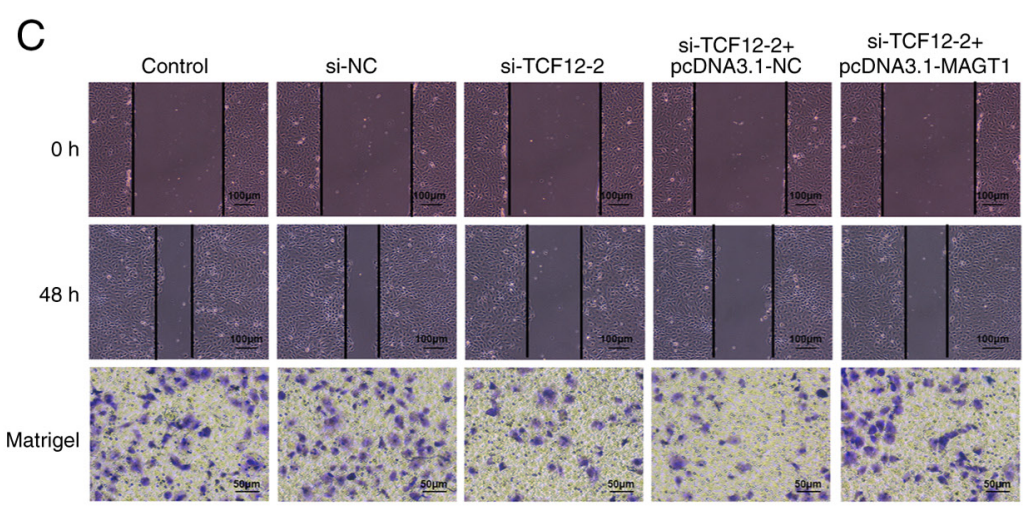
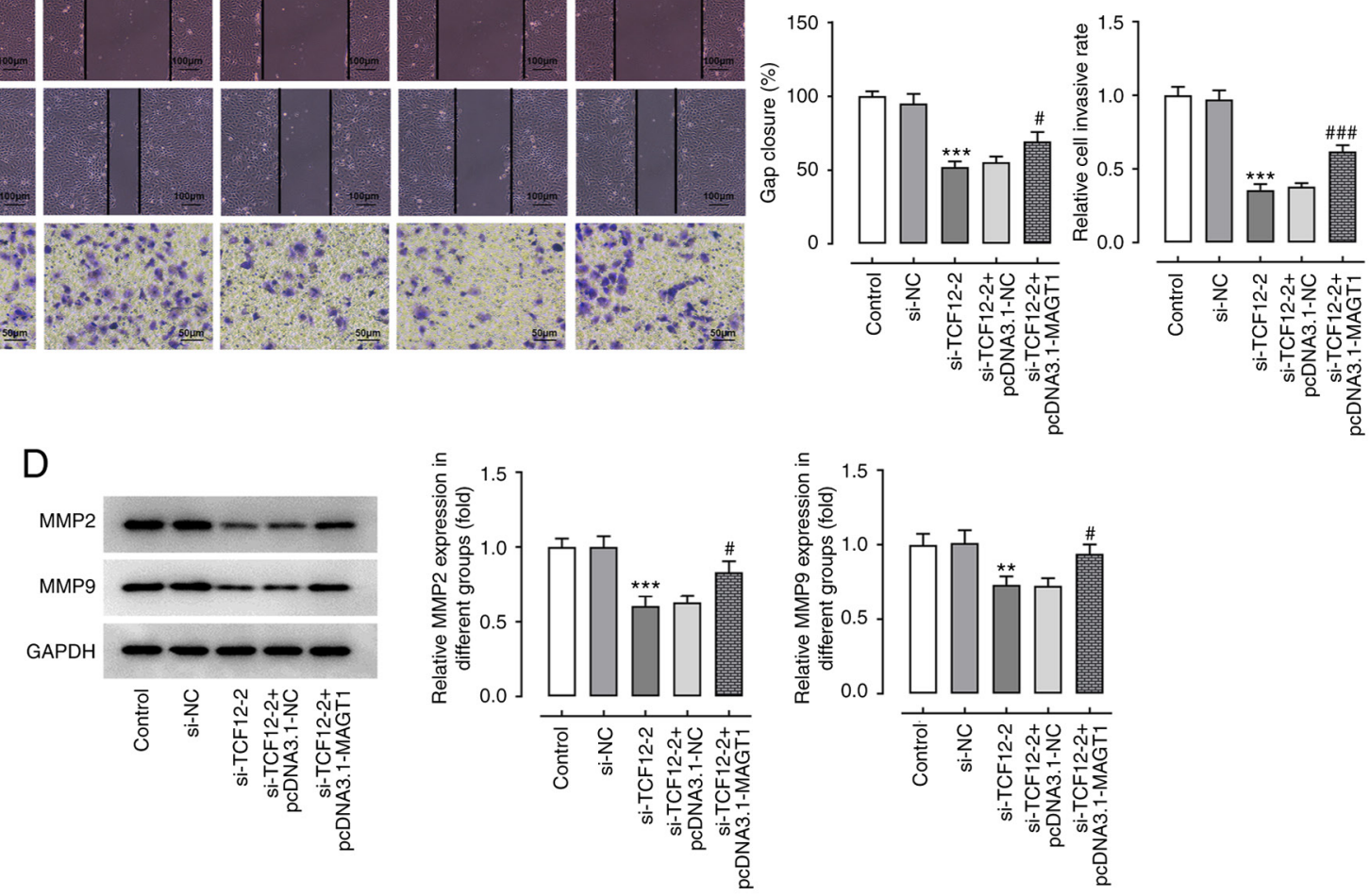

Figure 6. TCF12 promotes the proliferation, migration and invasion of pancreatic carcinoma cells by regulating the expression of MAGT1.(A) Cell Counting Kit- 8 assay was used to determine the cell viability in cells transfected with si-NC, si-TCF12-2, si-TCF12-2+pcDNA3.1-NC and si-TCF12-2+pcDNA3.1-MAGT1-2 at different time points (24, 48 and $72 \mathrm{~h}$. (B) Protein and mRNA expression levels of Ki67, and PCNA in BxPC-3 cells were decreased in cells transfected with si-TCF12-2 compared with that in cells transfected with si-NC group. Overexpression of MAGT1 reversed the protein expression level of Ki67 and PCNA. GAPDH served as the loading control. (C) The BxPC-3 cells transfected with si-TCF12-2 had decreased migration and invasion compared with that in cells transfected with si-NC. Overexpression of MAGT1 reversed the decreased migration and invasion. (D) Protein and mRNA expression level of MMP2, and MMP9 was significantly decreased in the BxPC-3 cells transfected with si-TCF12-2 compared with that in cells transfected with si-NC. The expression level of MMP2 and MMP9 were partly increased when co-transfected with pcDNA3.1-MAGT1, and si-TCF12-2. ${ }^{* *} \mathrm{P}<0.01,{ }^{* * * *} \mathrm{P}<0.001$ vs. si-NC group; ${ }^{\#} \mathrm{P}<0.05,{ }^{\# \# \#} \mathrm{P}<0.001$ vs. si-TCF12-2 + pcDNA3.1-NC group. NC, negative control; TCF12, transcription factor 12; MMP2, matrix metallopeptidase 2; si, small interfering.

is necessary for vertebrate embryo development (28). The role of MAGT1 in tumorigenesis seems to be two-sided and its absence has been associated with impaired $\mathrm{T}$ cell activation, and increased risk of hematological malignancies $(29,30)$. In addition, the low mRNA expression level of MAGT1 in ovarian serous carcinomas has been associated with poor prognosis (31). It has also been shown that overexpression of MAGT1 in other types of cancer, such as colorectal cancer and glioma, promotes the occurrence of cancer (9-13). Based on the data downloaded from GEPIA, it was found that MAGT1 mRNA expression level was significantly higher in the tumor tissues from patients with PC and its high expression level was associated with poor prognosis. Zheng et al (13) stated that the high mRNA expression level of MAGT1 in colorectal cancer also indicated a poor prognosis. Notably, magnesium transporters have been shown to play an important role in digestive cancer (32). In addition, the magnesium transporter protein solute carrier family 41 member 1 was reported to suppress human pancreatic ductal adenocarcinoma (33). In the present study, the high expression level of MAGT1 was also found in PC. Therefore, we hypothesized that MAGT1 could be an oncogene in PC. Subsequently, it was found that 
MAGT1 promoted the proliferation, migration and invasion of PC cells following overexpression or knockdown of MAGT1 expression in vitro. This is consistent with the proliferation, migration and invasion effects found in glioma, and colorectal cancer $(12,13)$.

The role of TCF12, as an upstream molecule of MAGT1, was also investigated in the development of PC. According to the results from the ENCORI website, the mRNA expression levels of TCF12 and MAGT1 were positively correlated in tissues from patients with PC. To the best of our knowledge, the function of TCF12 has not been investigated in PC; however, studies in hepatocellular carcinoma, gallbladder cancer, gastric cancer, myeloid tumors and prostate cancer have showed that TCF12 plays an oncogenic role (15-24). In the present study, the role of TCF12 was investigated PC. According to the data from GEPIA, the high expression level of TCF12 was also associated with poor overall survival in patients with PC, suggesting that MAGT1 and TCF12 could play oncogenic roles in PC. Then, following overexpression or knockdown of TCF12 expression, there were also significant changes in the protein and mRNA expression levels of MAGT1. This suggests that TCF12 may be upstream of MAGT1. Next, a dual-luciferase gene reporter assay and ChIP experiments revealed that TCF12 could bind to the promoter regions $(-1,932$ to $-1,922$ and -162 to -152$)$ upstream of the MAGT1 transcription start site to activate MAGT1 expression. The following experiments verified that knockdown of TCF12 expression could inhibit the proliferation, migration and invasion of PC cells in vitro, and these phenomena were reversed following overexpression of MAGT1.

In summary, the mRNA expression levels of MAGT1 and TCF12 were significantly increased in the tumor tissues of patients with PC, and several cell lines of PC. The upregulation of MAGT1 and TCF12 was also associated with poor prognosis. Furthermore, TCF12 activated the expression level of MAGT1 and promoted the proliferation, migration, and invasion of PC cells in vitro. The results provide a possible and theoretical basis for the use of MAGT1 as a clinical prognostic indicator of PC. However, the signaling pathway involved in the TCF12/MAGT1 axis has not been analyzed, which will be investigated in future experiments. In addition, the effect of the TCF12/MAGT1 axis on the development of PC in tumor-bearing mice will also be investigated. Furthermore, other PC cell lines will be used and additional PC malignant processes, such as cell cycle and apoptosis will be analyzed to further confirm the results from the present study.

\section{Acknowledgements}

Not applicable.

\section{Funding}

No funding was received.

\section{Availability of data and materials}

The datasets used and/or analyzed during the current study are available from the corresponding author on reasonable request.

\section{Authors' contributions}

GQS guided the project. LW and GQS conceived the study, analyzed the data and wrote the manuscript. LW, YJT and HYW performed the experiments. GQS and LW confirm the authenticity of all raw data. All authors read and approved the final manuscript.

\section{Ethics approval and consent to participate}

Not applicable.

\section{Patient consent for publication}

Not applicable.

\section{Competing interests}

The authors declare that they have no competing interests.

\section{References}

1. Siegel RL, Miller KD and Jemal A: Cancer statistics, 2018. CA Cancer J Clin 68: 7-30, 2018.

2. Rahib L, Smith BD, Aizenberg R, Rosenzweig AB, Fleshman JM and Matrisian LM: Projecting cancer incidence and deaths to 2030: The unexpected burden of thyroid, liver, and pancreas cancers in the United States. Cancer Res 74: 2913-2921, 2014.

3. Ryan DP, Hong TS and Bardeesy N: Pancreatic adenocarcinoma. N Engl J Med 371: 1039-1049, 2014.

4. Kleeff J, Korc M, Apte M, La Vecchia C, Johnson CD, Biankin AV, Neale RE, Tempero M, Tuveson DA, Hruban RH and Neoptolemos JP: Pancreatic cancer. Nat Rev Dis Primers 2: $16022,2016$.

5. Dougan SK: The pancreatic cancer microenvironment. Cancer J 23: 321-325, 2017.

6. Lei MZ, Li XX, Zhang Y, Li JT, Zhang F, Wang YP, Yin M, Qu J and Lei QY: Acetylation promotes BCAT2 degradation to suppress BCAA catabolism and pancreatic cancer growth. Signal Transduct Target Ther 5: 70, 2020.

7. Quamme GA: Molecular identification of ancient and modern mammalian magnesium transporters. Am J Physiol Cell Physiol 298: C407-C429, 2010.

8. Chaigne-Delalande B, Li FY, O'Connor GM, Lukacs MJ, Jiang P, Zheng L, Shatzer A, Biancalana M, Pittaluga S, Matthews $\mathrm{HF}$, et al: $\mathrm{Mg} 2+$ regulates cytotoxic functions of NK and CD8 T cells in chronic EBV infection through NKG2D. Science 341: 186-191, 2013.

9. Cazzaniga A, Moscheni C, Trapani V, Wolf FI, Farruggia G, Sargenti A, Lotti S, Maier J and Castiglioni S: The different expression of TRPM7 and MagT1 impacts on the proliferation of colon carcinoma cells sensitive or resistant to doxorubicin. Sci Rep 7: 40538, 2017.

10. Li H, Xia T, Guan Y and Yu Y: Sevoflurane regulates glioma progression by Circ_0002755/miR-628-5p/MAGT1 axis. Cancer Manag Res 12: 5085-5098, 2020.

11. Molee P, Adisakwattana P, Reamtong O, Petmitr S, Sricharunrat T, Suwandittakul N and Chaisri U: Up-regulation of AKAP13 and MAGT1 on cytoplasmic membrane in progressive hepatocellular carcinoma: A novel target for prognosis. Int J Clin Exp Pathol 8: 9796-9811, 2015.

12. Wang G, Li Y, Li J, Zhang D, Luo C, Zhang B and Sun X: microRNA-199a-5p suppresses glioma progression by inhibiting MAGT1. J Cell Biochem 120: 15248-15254, 2019.

13. Zheng K, Yang Q, Xie L, Qiu Z, Huang Y, Lin Y, Tu L and Cui C: Overexpression of MAGT1 is associated with aggressiveness and poor prognosis of colorectal cancer. Oncol Lett 18: 3857-3862, 2019.

14. Zheng L, Xue J, Jaffee EM and Habtezion A: Role of immune cells and immune-based therapies in pancreatitis and pancreatic ductal adenocarcinoma. Gastroenterology 144: 1230-1240, 2013. 
15. Chen QB, Liang YK, Zhang YQ, Jiang MY, Han ZD, Liang YX, Wan YP, Yin J, He HC and Zhong W: Decreased expression of TCF12 contributes to progression and predicts biochemical recurrence in patients with prostate cancer. Tumour Biol 39: 1010428317703924,2017

16. Cao J, Wei J, Yang P, Zhang T, Chen Z, He F, Wei F, Chen H, Hu H, Zhong J, et al: Genome-scale CRISPR-Cas9 knockout screening in gastrointestinal stromal tumor with Imatinib resistance. Mol Cancer 17: 121, 2018.

17. L'Abbate A, Tolomeo D, De Astis F, Lonoce A, Lo Cunsolo C Mühlematter D, Schoumans J, Vandenberghe P, Van Hoof A, Palumbo $\mathrm{O}$, et al: $\mathrm{t}(15 ; 21)$ translocations leading to the concurrent downregulation of RUNX1 and its transcription factor partner genes SIN3A and TCF12 in myeloid disorders. Mol Cancer 14: 211,2015

18. Gao S, Bian T, Zhang Y, Su M and Liu Y: TCF12 overexpression as a poor prognostic factor in ovarian cancer. Pathol Res Pract 215: 152527, 2019

19. Lee CC, Chen WS, Chen CC, Chen LL, Lin YS, Fan CS and Huang TS: TCF12 protein functions as transcriptional repressor of E-cadherin, and its overexpression is correlated with metastasis of colorectal cancer. J Biol Chem 287: 2798-2809, 2012.

20. Chen WS, Chen CC, Chen LL, Lee CC and Huang TS Secreted heat shock protein $90 \alpha$ (HSP90 $\alpha$ ) induces nuclear factor- $\mathrm{kB}$-mediated TCF12 protein expression to down-regulate E-cadherin and to enhance colorectal cancer cell migration and invasion. J Biol Chem 288: 9001-9010, 2013.

21. Yang J, Zhang L, Jiang Z, Ge C, Zhao F, Jiang J, Tian H, Chen T, $\mathrm{Xie} \mathrm{H}$, Cui Y, et al: TCF12 promotes the tumorigenesis and metastasis of hepatocellular carcinoma via upregulation of CXCR4 expression. Theranostics 9: 5810-5827, 2019.

22. Wang X, Gao S, Xie F, Li W, Li M, Yan N, Gao T and Fang L: High expression of TCF12 contributes to gastric cancer development via being target regulated by miR-183 and activating PI3K/AKT pathway. J Cell Biochem 120: 13903-13911, 2019.

23. He J, Shen S, Lu W, Zhou Y, Hou Y, Zhang Y, Jiang Y, Liu H and Shao Y: HDAC1 promoted migration and invasion binding with TCF12 by promoting EMT progress in gallbladder cancer. Oncotarget 7: 32754-32764, 2016.

24. Luo C, Balsa E, Perry EA, Liang J, Tavares CD, Vazquez F, Widlund HR and Puigserver P: H3K27me3-mediated PGC10 gene silencing promotes melanoma invasion through WNT5A and YAP. J Clin Invest 130: 853-862, 2020.
25. Livak KJ and Schmittgen TD: Analysis of relative gene expression data using real-time quantitative PCR and the 2(-Delta Delta C(T)) method. Methods 25: 402-408, 2001

26. Si L, Fu J, Liu W, Hayashi T, Nie Y, Mizuno K, Hattori S, Fujisaki H, Onodera S and Ikejima T: Silibinin inhibits migration and invasion of breast cancer MDA-MB-231 cells through induction of mitochondrial fusion. Mol Cell Biochem 463: 189-201, 2020.

27. Gobin E, Bagwell K, Wagner J, Mysona D, Sandirasegarane S, Smith N, Bai S, Sharma A, Schleifer R and She JX: A pan-cancer perspective of matrix metalloproteases (MMP) gene expression profile and their diagnostic/prognostic potential. BMC Cancer 19: 581, 2019.

28. Zhou H and Clapham DE: Mammalian MagT1 and TUSC3 are required for cellular magnesium uptake and vertebrate embryonic development. Proc Natl Acad Sci USA 106: 15750-15755, 2009.

29. Gile J, Ruan G, Abeykoon J, McMahon MM and Witzig T: Magnesium: The overlooked electrolyte in blood cancers? Blood Rev 44: 100676, 2020.

30. Ravell J, Chaigne-Delalande B and Lenardo M: X-linked immunodeficiency with magnesium defect, Epstein-Barr virus infection, and neoplasia disease: A combined immune deficiency with magnesium defect. Curr Opin Pediatr 26: 713-719, 2014.

31. Willis S, Villalobos VM, Gevaert O, Abramovitz M, Williams C, Sikic BI and Leyland-Jones B: Single gene prognostic biomarkers in ovarian cancer: A meta-analysis. PLoS One 11: e0149183, 2016.

32. Auwercx J, Rybarczyk P, Kischel P, Dhennin-Duthille I, Chatelain D, Sevestre H, Van Seuningen I, Ouadid-Ahidouch H, Jonckheere $\mathrm{N}$ and Gautier $\mathrm{M}: \mathrm{Mg}(2+)$ transporters in digestive cancers. Nutrients 13: 210, 2021.

33. Xie J, Cheng CS, Zhu XY, Shen YH, Song LB, Chen H, Chen Z, Liu LM and Meng ZQ: Magnesium transporter protein solute carrier family 41 member 1 suppresses human pancreatic ductal adenocarcinoma through magnesium-dependent Akt/mTOR inhibition and bax-associated mitochondrial apoptosis. Aging (Albany NY) 11: 2681-2698, 2019.

This work is licensed under a Creative Commons Attribution-NonCommercial-NoDerivatives 4.0 International (CC BY-NC-ND 4.0) License. 